1. Ooi, S.K., O'Donnell, A.H. \& Bestor, T.H. J. Cell Sci. 122, 2787-2791 (2009).

2. Tahiliani, M. et al. Science 324, 930-935 (2009).

3. Ito, S. et al. Science 333, 1300-1303 (2011).

4. He, Y.F. et al. Science 333, 1303-1307 (2011).

5. Inoue, A., Shen, L., Dai, Q., He, C. \& Zhang, Y. Cell Res. 21, 1670-1676 (2011).

6. Inoue, A. \& Zhang, Y. Science 334, 194 (2011).

7. Nabel, C.S. \& Kohli, R.M. Science 333, 1229-1230 (2011).

8. Kellinger, M.W. et al. Nat. Struct. Mol. Biol. 19, 831-833 (2012).

9. Aoki, F., Worrad, D.M. \& Schultz, R.M. Dev. Biol. 181 296-307 (1997).

10. van Luenen, H.G. et al. Cell 150, 909-921 (2012).

11. Borst, P. \& Sabatini, R. Annu. Rev. Microbiol. 62, 235-251 (2008).
12. Iyer, L.M., Tahiliani, M., Rao, A. \& Aravind, L. Cell Cycle 8, 1698-1710 (2009).

13. Iyer, L.M., Abhiman, S. \& Aravind, L. Prog. Mol. Biol. Transl. Sci. 101, 25-104 (2011).

14. Gommers-Ampt, J.H. \& Borst, P. FASEB J. 9, 1034-1042 (1995).

15. van Leeuwen, F. et al. Genes Dev. 11, 3232-3241 (1997).

16. Murata-Kamiya, N. et al. Nucleic Acids Res. 27, 4385-4390 (1999).

17. Kamiya, H. et al. J. Biochem. 132, 551-555 (2002).

18. Maiti, A. \& Drohat, A.C. J. Biol. Chem. 286, 35334-35338 (2011).

19. Zhang, L. et al. Nat. Chem. Biol. 8, 328-330 (2012).

20. Nabel, C.S. et al. Nat. Chem. Biol. 8, 751-758 (2012).

21. Williams, K. et al. Nature 473, 343-348 (2011).

22. Wu, H. et al. Genes Dev. 25, 679-684 (2011).
23. Huang, Y., Pastor, W.A., Zepeda-Martinez, J.A. \& Rao, A. Nat. Protoc. 7, 1897-1908 (2012).

24. Pastor, W.A., Huang, Y., Henderson, H.R., Agarwal, S. \& Rao, A. Nat. Protoc. 7, 1909-1917 (2012).

25. Pastor, W.A. et al. Nature 473, 394-397 (2011)

26. Ficz, G. et al. Nature 473, 398-402 (2011).

27. Song, C.X. et al. Nat. Methods 9, 75-77 (2011).

28. Booth, M.J. et al. Science 336, 934-937 (2012)

29. Yu, M. et al. Cell 149, 1368-1380 (2012).

30. Raiber, E.A. et al. Genome Biol. 13, R69 (2012).

31. Schiesser, S. et al. Angew. Chem. Int. Edn. Engl. 51, 6516-6520 (2012).

32. Cortázar, D., Kunz, C., Saito, Y., Steinacher, R. \& Schar, P. DNA Repair (Amst.) 6, 489-504 (2007).

33. Kallin, E.M. et al. Mol. Cell published online, doi:10.1016/j.molcel.2012.08.007 (13 September 2012).

34. Shukla, S. et al. Nature 479, 74-79 (2011).

\title{
Asymmetrically modified nucleosomes expand the histone code
}

\author{
Barth van Rossum, Wolfgang Fischle \& Philipp Selenko
}

\begin{abstract}
According to the 'classical' view, histone modifications are established in an identical fashion on both copies of each of the four core histones, resulting in 'symmetrically' modified nucleosomes. Now, a new study challenges this notion by demonstrating that asymmetric histone modifications exist on individual mononucleosomes in native chromatin and that symmetric and asymmetric modifications signal different biological outcomes, suggesting a radically expanded histone code.
\end{abstract}

The epigenetic landscape is shaped by differentially methylated DNA and a large repertoire of histone modifications that alter the physicochemical properties of nucleosomes, the basic building blocks of higher-order chromatin. Each nucleosome contains two identical copies of the core histones, $\mathrm{H} 2 \mathrm{~A}$, $\mathrm{H} 2 \mathrm{~B}, \mathrm{H} 3$ and $\mathrm{H} 4$, which assemble into the protein octamer that provides the architectural basis for packaging chromosomal DNA $^{1}$. This two-copy arrangement also creates the possibility for functional redundancy with respect to post-translational histone modifications (PTMs), which have long been known to define chromosomal regions of high (euchromatin) and low (heterochromatin) transcriptional activity ${ }^{2}$. The current notion suggests that histone modifications occur in a symmetrical fashion on individual mononucleosomes, meaning that identical

Barth van Rossum and Philipp Selenko are at the Leibniz Institute of Molecular Pharmacology (FMP Berlin), Department of NMR-supported Structural Biology, Berlin, Germany. Wolfgang Fischle is at the Chromatin Biochemistry Laboratory, Max Planck Institute for Biophysical Chemistry, Göttingen, Germany. e-mail: selenko@fmp-berlin.de positions in both sister histones are modified in exactly the same way. This concept is well established because, in the context of chromatin, enzymes that modify nucleosomal histones encounter sequence-identical substrate moieties in close proximity, with few features that would allow discrimination by the enzymes.

However, reporting in Cell, Voigt et al. ${ }^{3}$ used micrococcal nuclease-digested chromosomal mononucleosomes from embryonic stem cells, mouse embryonic fibroblasts and cultured HeLa cells, in combination with high-specificity antibody affinity purification and quantitative mass spectrometry (LC-MS/ MS), to show that histone H3 Lys27 di- and trimethylation marks (H3K27me2/3) occur both symmetrically and asymmetrically in native chromatin, in approximately equal proportions (Fig. 1a). Surprisingly, in mononucleosomes that contained $\mathrm{H} 3 \mathrm{~K} 27 \mathrm{me} 2 / 3$ on one sister histone, the respective other $\mathrm{H} 3$ copy often harbored different methylation marks, either trimethylated Lys4 (H3K4me3) or di- and trimethylated Lys36 (H3K36me2/3) (Fig. 1b). These sets of methylation marks also constitute so-called bivalent chromatin domains, which denote the joint occurrence of activating (that is, $\mathrm{H} 3 \mathrm{~K} 4 \mathrm{me} 3, \mathrm{H} 3 \mathrm{~K} 36 \mathrm{me} 2 / 3$ ) and repressing (that is, H3K27me2/3) PTM signals that are often found in poised promoter regions ${ }^{4}$. Similarly, another repressive chromatin mark, monomethylated histone H4 Lys20 (H4K20me1), was also shown to occur in a symmetric and asymmetric fashion in native chromatin. When recombinant nucleosomes containing symmetric H3K27me3, H3K4me3 or H3K36me3 were mixed with Polycomb repressive complex 2 (PRC2), no further methylation was observed $^{5}$. In contrast, asymmetrically modified nucleosomes were efficiently methylated on the 'vacant' H3 sister histone (Fig. 1b). Thereby, asymmetrically and differentially modified nucleosomes were created, thus providing a rationale for the establishment of bivalent chromatin domains.

The notion of asymmetrically modified nucleosomes dramatically expands the combinatorial space of independent sets of histone modifications. According to the classical view, every nucleosome carries $2^{n}$ possibilities (per histone) to signal different functional states, with $n$ being the number of possible modifications. The occurrence of asymmetric histone modifications expands this space by the power of 2 to $\left(2^{n}\right)^{2}$. If we assume that asymmetric histone modifications can 
a

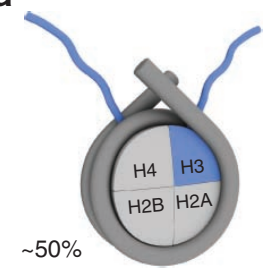

Unmodified

b

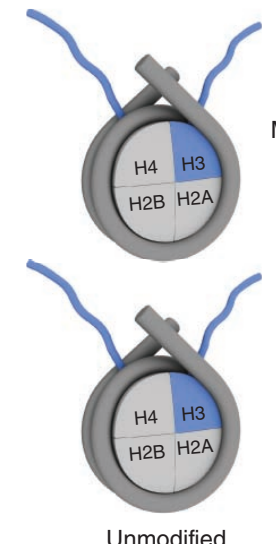

Unmodified

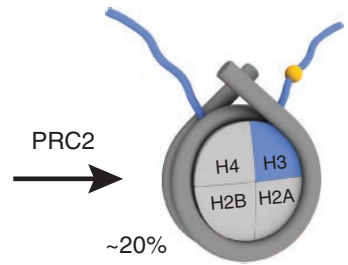

Asymmetric

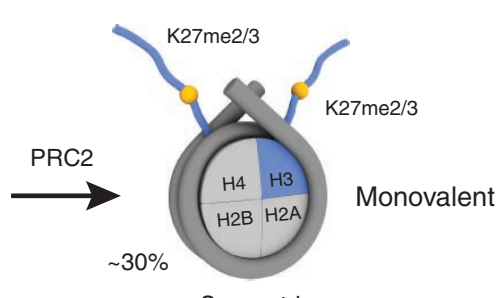

Symmetric

Figure 1 Reaction models for the generation of symmetrically and asymmetrically modified nucleosomes. (a) Average populations of unmodified and asymmetrically and symmetrically modified di- and trimethylated histone H3 Lys27 (K27me2/3) nucleosomes found in different cell lines and the proposed reaction model for their generation by PRC2 (ref. 3). (b) Hypothetical MLL-SET1 and SET2 reactions for the in vivo establishment of asymmetric methylation marks on H3 Lys4 (K4me3) and Lys36 (K36me3). In vitro reactions of asymmetrically modified nucleosomes with PRC2-produced asymmetric bivalent chromatin marks.

independently occur on all four core histones, this number grows to $\left(2^{n}\right)^{2 \times 4}$ for every nucleosome and quickly reaches vast proportions when extended to nucleosomal arrays. Clearly, this theoretical space will never be physically explored, owing to modification cross-talk and de facto interdependence of most histone PTM states. As Voigt et al. ${ }^{3}$ have shown, negative modification cross-talk between sister histones may not even allow the expansion by a factor of 2 . Nonetheless, asymmetric modification marks add a layer of complexity that raises important mechanistic and functional questions.

For example, how are asymmetric modification marks laid down in the first place? Probably the simplest model is a single initial modification event that stochastically affects individual substrate sites while leaving many sites unreacted (that is, incomplete substrate turnover) (Fig. 2a). On a topological chromatin scale, this would result in a random number of asymmetric modification marks that may function as seeds to further propagate asymmetric PTM states through negativeor positive-modification cross-talk that can occur within individual nucleosomes (intra) or between neighboring nucleosomes (inter) ${ }^{6}$. Voigt et al. ${ }^{3}$ applied a similar mathematical reaction model to explain the relative abundances of asymmetrically and symmetrically modified nucleosomes in their experimental data sets (Fig. 1a). There are two problems with such theoretical models. First, under steady-state conditions (that is, given enough time and provided that there is sustained enzyme activity and no demodification), most substrate molecules would eventually end up in the symmetric product state. Second, histone-modifying enzymes are unlikely to act in isolation, so that any consecutive reaction model falls short in acknowledging the highly parallel nature of most cellular processes. More realistically, especially in the context of native, dense chromatin ${ }^{7}$, multiple histonemodifying enzymes act locally in a simultaneous and stochastic manner to establish individual PTM marks, whose presence on one sister histone may negatively influence the establishment of the respective other modification mark (intranucleosomal crosstalk). In such a model, enzymes 'compete' for histone-modification sites according to their local abundance and intrinsic activities.
Once a sister histone has been 'marked' with a certain PTM, it is excluded from modification by the other enzyme. In fact, the topology of higher-order chromatin may exhibit few discriminatory features to enable a distinct source-of-origin separation of sister histones within the same nucleosome and of histones in neighboring nucleosomes (Fig. 2a). In the case of asymmetric $\mathrm{H} 3 \mathrm{~K} 27 \mathrm{me} 2 / 3$ and $\mathrm{H} 3 \mathrm{~K} 4 \mathrm{me} 3$ or $\mathrm{H} 3 \mathrm{~K} 36 \mathrm{me} 2 / 3$ modification, PRC2 and members of the MLL-SET1 (ref. 8) and SET2 (ref. 9) families of methyltransferases could jointly be involved in such modification reactions, which is supported by the in vitro PRC2 data reported by Voigt et al. ${ }^{3}$.

Another way of establishing asymmetric histone modifications was recently described in our own work ${ }^{10}$. Using time-resolved, high-resolution NMR spectroscopy, Liokatis et al. showed that two histone-modifying kinases, checkpoint kinase 1 (Chk1) and protein kinase $\mathrm{C}(\mathrm{PKC})$, displayed dual substrate site specificities on histone $\mathrm{H} 3$ tails that led to different phosphorylation marks on individual $\mathrm{H} 3$ molecules (Fig. 2b). Both of these marks also functioned as intramolecular inhibitors of the respective other modification event so that they occurred in a mutually exclusive fashion on individual $\mathrm{H} 3$ tails. By means of these actions, both enzymes established different asymmetric histone marks in single modification reactions, which may similarly expand the mixed PTM topology of the epigenetic landscape (Fig. 2a).

What are the functional consequences of asymmetric nucleosome modifications? As Voigt et al. ${ }^{3}$ have laid out, bivalent chromatin domains (that is, functionally antagonizing PTM marks) could help maintain poised transcription states of individual genes. Removal of one of these marks may be sufficient to induce transcriptional activation or repression, thereby alleviating the requirements for exhaustive modifications and ensuring that fast changes in cellular behaviors can be executed in response to external or internal cues. Interestingly, Voigt et al. ${ }^{3}$ find no apparent differences in the proportions of H3K27me2/3 symmetrically and asymmetrically modified nucleosomes upon embryonic stem cell differentiation with retinoic acid, despite increased levels of other repressive PTM marks such as di- and trimethylated H3 Lys9 (H3K9me2/3) and trimethylated H4 Lys20 (H4K20me3).

In addition, asymmetrically modified nucleosomes may provide altered binding surfaces for 'tethered' chromatin-remodeling complexes, and multivalent recognition of differentially modified histone tails may offer new means to accommodate 
a

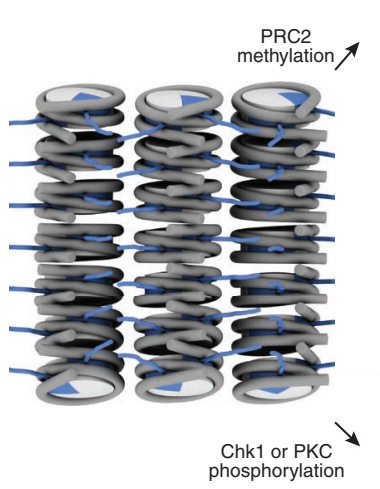

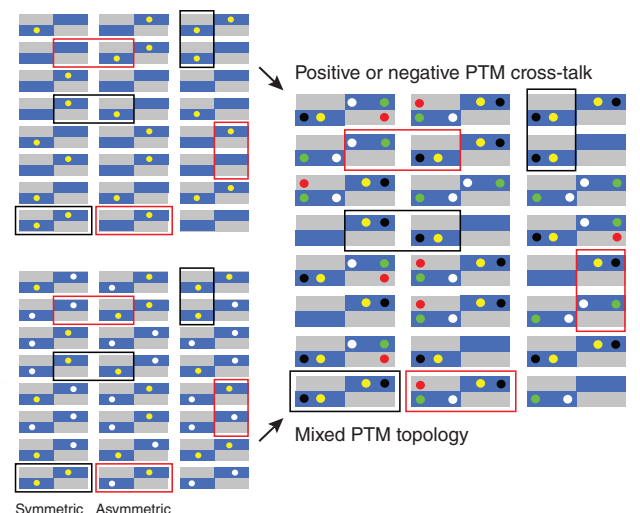

b
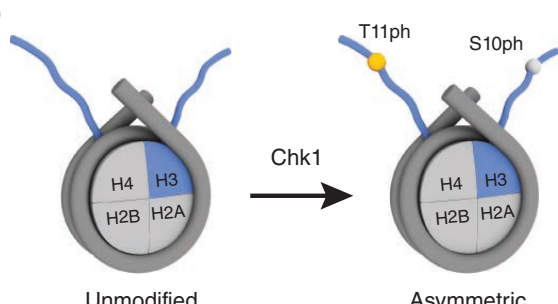

Asymmetric

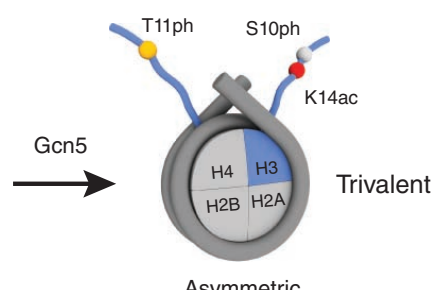

Asymmetric
C

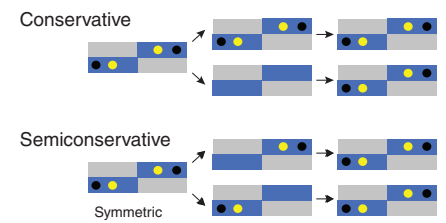

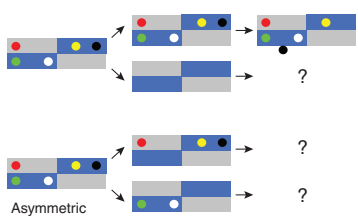

Figure 2 Complex PTM topologies in higher-order chromatin. (a) Illustration of the hypothetical level of structural degeneracy of $\mathrm{N}$-terminal histone $\mathrm{H} 3$ tails in higher-order chromatin (left). Schematic depiction of the same topology and stochastic modifications of single histone sites in a symmetric (black box) and asymmetric (red box) fashion, according to the proposed reaction model for PRC2 (ref. 3) (top middle). In such an environment, topological levels of symmetry and asymmetry may be relevant within individual nucleosomes (intranucleosomal) but also between neighboring nucleosomes (internucleosomal). Additional modifications through positive and negative cross-talk of other enzymes may lead to the mosaic appearance of asymmetrically modified nucleosome arrays (right). Joint establishment of mutually exclusive asymmetric histone-modification marks are illustrated according to the proposed reaction model for Chk1 (ref. 10) (bottom middle). Further modification cross-talk may similarly establish mixed PTM topologies. (b) Reaction model for the generation of asymmetric H3 Thr11 (T11ph)- and Ser10 (S10ph)phosphorylated nucleosomes by Chk1 and further modification to K14ac by the histone acetyltransferase Gcn5 (ref. 10). (c) Reaction models for the segregation of symmetrically and asymmetrically modified nucleosomes during DNA replication. Whereas symmetrically modified nucleosomes can faithfully transmit their modification states along the conservative and semiconservative routes, how this is achieved in asymmetrically modified nucleosomes remains to be determined. The color coding of the histone marks is as in Figure 1 (yellow, K27me2/3; white, K36me3), and green, red and black indicate other unspecified histone marks.

intra- and internucleosomal bridging interactions $^{11}$. The occurrence of symmetric or asymmetric modification patterns may further fine-tune the 'spatial' affinities of individual chromatin-binding domains and thereby regulate global recruitment processes ${ }^{12}$.

How are asymmetrically modified nucleosomes segregated during DNA replication? Voigt et al. ${ }^{3}$ rightfully point out that the semiconservative model of $\mathrm{H} 3-\mathrm{H} 4$ dimer segregation breaks down in the face of asymmetrically modified nucleosomes (Fig. 2c), as it requires symmetrically modified sister histones for the faithful transmission and inheritance of epigenetic memory ${ }^{13}$. Even if the semiconservative model of $\mathrm{H} 3-\mathrm{H} 4$ dimer segregation is not widespread, as a recent study suggests ${ }^{14}$, how is the reestablishment of asymmetrically modified nucleosomes accomplished after DNA replication? Is it a regulated process, or does it again involve stochastic modification reactions? The data by Voigt et al. ${ }^{3}$ support the notion of conserved maintenance of nucleosome asymmetry over multiple rounds of cell division, but how is this achieved?

As so often occurs, the data by Voigt et $a l .^{3}$ raise a multitude of additional questions. The question posed by Liokatis et al. ${ }^{10}$ as to whether asymmetrically modified nucleosomes occur in a chromosomal context has now been answered, but many more remain to be addressed.

\section{COMPETING FINANCIAL INTERESTS}

The authors declare no competing financial interests.

1. Luger, K., Mader, A.W., Richmond, R.K., Sargent, D.F. \& Richmond, T.J. Nature 389, 251-260 (1997).

2. Jenuwein, T. \& Allis, C.D. Science 293, 1074-1080 (2001).

3. Voigt, P. et al. Cell 151, 181-193 (2012).

4. Bernstein, B.E. et al. Cell 125, 315-326 (2006).

5. Schmitges, F.W. et al. Mol. Cell 42, 330-341 (2011).

6. Latham, J.A. \& Dent, S.Y. Nat. Struct. Mol. Biol. 14 1017-1024 (2007).

7. Yuan, W. et al. Science 337, 971-975 (2012).

8. Santos-Rosa, $\mathrm{H}$. et al. Nature $419,407-411$ (2002).

9. Venkatesh, S. et al. Nature 489, 452-455 (2012).

10. Liokatis, S. et al. Nat. Struct. Mol. Biol. 19, 819-823 (2012).

11. Ruthenburg, A.J. et al. Cell 145, 692-706 (2011).

12. Taverna, S.D., Li, H., Ruthenburg, A.J., Allis, C.D. \& Patel, D.J. Nat. Struct. Mol. Biol. 14, 1025-1040 (2007).

13. Ransom, M., Dennehey, B.K. \& Tyler, J.K. Cell 140 183-195 (2010).

14. Xu, M. et al. Science 328, 94-98 (2010). 